\title{
Laboreal
}

Volume $2 \mathrm{~N}^{\circ} 2$ | 2006

Varia

\section{Aportes del trabajo colectivo a la gestion de la seguridad laboral en situación de riesgo en el àmbito ferroviario}

Contribuições do trabalho colectivo para a gestão da segurança do trabalho em situações de risco no sector ferroviário

Apports du travail collectif à la gestion de la sécurité au travail en situation de risque dans le secteur ferroviaire

Contributions of collective work to the management of occupational safety in hazardous situations in the railway domain

\section{Cecília De La Garza y Annie Weill-Fassina}

\section{(2) OpenEdition}

\section{Journals}

Edición electrónica

URL: http://journals.openedition.org/laboreal/13274

ISSN: 1646-5237

\section{Editor}

Universidade do Porto

\section{Referencia electrónica}

Cecília De La Garza y Annie Weill-Fassina, « Aportes del trabajo colectivo a la gestion de la seguridad laboral en situación de riesgo en el àmbito ferroviario », Laboreal [En línea], Volume 2 N² | 2006

Publicado el 01 diciembre 2006, consultado el 10 octubre 2019. URL : http://journals.openedition.org/ laboreal/13274

Este documento fue generado automáticamente el 10 octubre 2019.

Laboreal está licenciado com uma Licença Creative Commons - Atribuição-NãoComercial 4.0 Internacional. 


\section{Aportes del trabajo colectivo a la gestion de la seguridad laboral en situación de riesgo en el àmbito ferroviario}

Contribuições do trabalho colectivo para a gestão da segurança do trabalho em situações de risco no sector ferroviário

Apports du travail collectif à la gestion de la sécurité au travail en situation de risque dans le secteur ferroviaire

Contributions of collective work to the management of occupational safety in hazardous situations in the railway domain

Cecília De La Garza y Annie Weill-Fassina

\section{NOTA DEL EDITOR}

Manuscrito recebido em : Setembro/2006

Aceite após peritagem em : Novembro/2006

\section{Introducción, contexto de los estudios ferroviarios}

El objetivo de éste artículo es el de presentar una síntesis de tres estudios principales sobre el trabajo colectivo como un elemento importante en la gestión de los riesgos y de la seguridad. Estos estudios fueron realizados en el ámbito ferroviario por medio de contratos de investigación entre la Société Nationale des Chemins de Fer (SNCF), Compañía de Ferrocarriles Francesa, y distintos organismos de investigación. En la primera parte del artículo se presentará el marco teórico que guió dichos estudios y en 
la segunda y tercera partes se ilustrarán parte de los resultados y aportes principales del trabajo colectivo a la gestión de la seguridad.

Dicha compañía se enfrentaba con diferentes problemas de seguridad en contextos de alto riesgo. Estos se referían, por un lado, a accidentes laborales de los agentes de las vías que efectúan operaciones de mantenimiento y que están expuestos al llamado "riesgo de impacto" por circulación ferroviaria; por otro lado a un problema de seguridad del sistema socio-técnico en relación con las operaciones de mantenimiento de las instalaciones eléctricas que rigen el tráfico ferroviario. En el primer caso las consecuencias son en general mortales; en el segundo caso el riesgo es el de crear un incidente o accidente de circulación ferroviaria por ejemplo por una conexión eléctrica errónea que anunciará una luz verde en lugar de una luz roja, enviando así un tren hacia una vía ocupada por otro tren. Ambos estudios permitieron demostrar cómo, de maneras distintas, el trabajo colectivo es en cierto modo un criterio de seguridad y de fiabilidad del sistema socio-técnico (De la Garza, Maggi \& Weill-Fassina, 1999; De la Garza, 1999).

3 Existen medios de seguridad y de protección del personal que trabaja en las vías, pero el número de accidentes prueba que aún no se controlan todos los factores (entre $15 \mathrm{y}$ 20 por año). De la misma manera existen medios de protección y de control para las intervenciones y operaciones efectuadas en las instalaciones eléctricas. Sin embargo, los incidentes considerados como "contrarios a la seguridad ferroviaria" existen (entre 15 y 35 por año). No es la frecuencia del evento lo que preocupa tanto a la empresa como la gravedad de las consecuencias reales o probables. En el caso de los incidentes contrarios a la seguridad ferroviaria, el accidente no se produjo, pero si éste llegará a producirse las consecuencias serían catastróficas! Por ejemplo el encuentro frente e frente de dos trenes, el descarrilamiento de un tren, el atropellamiento de peatones o vehículos en un paso a nivel.

4 En ambos casos, el análisis ergonómico aporta un diagnóstico explicativo enfocando la gestión del riesgo a nivel de las interacciones entre los operadores, los riesgos cotidianos y los medios laborales. Tres métodos complementarios fueron utilizados para el estudio de los procesos accidentales e incidentales:

- la observación de campo de las actividades laborales individuales y colectivas para identificar los riesgos y dificultades típicos de estas situaciones de trabajo ;

- el análisis de reportes de accidentes e incidentes con un instrumento desarrollado para la empresa, la técnica de los "puntos pivote" [1] ;

- una serie de entrevistas individuales y de grupo con el fin de completar la comprensión del proceso laboral, así como la reconstitución de los eventos críticos.

Es así como se ponen en evidencia situaciones laborales en las cuales a pesar de la experiencia de los operadores, del trabajo colectivo y de los medios de seguridad existentes, los riesgos persisten. El objetivo final es entonces proponer recomendaciones para estas situaciones con el fin de favorecer el trabajo colectivo en situaciones laborales seguras y de contribuir a lo que varios autores definen como el "safety management" en las compañías (Baram, 1995 ; Hale \& Baram, 1998 ; Cox \& Cox, 1996). 


\subsection{El trabajo colectivo, prueba de seguridad}

El interés por el trabajo colectivo en ergonomía surge como una necesidad para poder analizar la actividad individual, y el marco organizacional en el cual dicha actividad se inscribe. El objetivo es entender los procesos de trabajo y las dificultades y riesgos que estos inducen. El análisis ergonómico permite la identificación de las incompatibilidades entre los requerimientos y restricciones laborales y las características de los operadores (aspectos cognitivos, experiencia, estado de salud o de fatiga, etc.), así como sus posibilidades o limites de gestión. El análisis ergonómico es un análisis de tipo "ascendente" descrito como "bottum up" en la literatura, en contraste con uno de tipo descendente dicho top down, que parte de la organización laboral (Hendrick, 1991; de Montmollin, 1992). Sin embargo adoptando un punto de vista global, es decir analizando las interacciones "hombremáquina", "hombre-entorno laboral", se consideran las relaciones entre las reglas estructurales elaboradas por la jerarquía y cubriendo las actividades de diseño del trabajo y las reglas operativas del proceso productivo desarrolladas durante la ejecución del trabajo por los operadores. El término "estructura" significa aquí la manera en que les partes de un conjunto concreto o abstracto son ordenadas entre ellas. La integración de esta dimensión social tiene como objetivo el entender los modos de relaciones que tejen los diferentes trabajadores, oficios, centros de decisión y niveles jerárquicos que participan a una gestión colectiva del trabajo y de la seguridad.

\subsection{El riesgo como objeto de "regulación"[2] de las interacciones entre las exigencias laborales y las características de los operadores}

7 El "riesgo" se aborda aquí en relación, no solo con la fiabilidad del sistema, sino también con la salud y la seguridad del operador. Se trata del riesgo en la actividad laboral o engendrado por dicha actividad. Éste se define en términos de posibilidad de ocurrencia de un evento no deseado más que en términos de probabilidad en la medida en que el propósito aquí no es el de cuantificar el riesgo sino identificar sus orígenes. El riesgo está ligado al proceso laboral y surge de la interacciones entre el estado funcional del operador y las características organizacionales, técnicas y del medio ambiente laboral, las cuales determinan las exigencias de las tareas. El riesgo surge y se actualiza durante la transformación del proceso laboral en proceso accidental o incidental. La identificación y la comprensión de los orígenes del riesgo laboral implica entonces el análisis de las modalidades de regulación de las situaciones que afrontan los trabajadores de manera cotidiana.

El análisis del trabajo colectivo intenta comprender los mecanismos de regulación y de compensación de las perturbaciones que se producen en la situación laboral. Vinculado con los procesos de trabajo el concepto de regulación tiene varios sentidos :

- Desde un punto de vista general, se refiere a la acción de regular, de asegurar un buen funcionamiento, como en física la graduación de una temperatura. El mantenimiento dinámico de una constante de las características deseadas a través de un sistema de retroacción ; éste modelo cibernético aplicándose a la fisiología como a la economía política.

- Desde el punto de vista de la organización laboral, se refiere al conjunto de reglas que definen el proceso de acciones et de decisiones (Maggi, 1996). 
- Desde el punto de vista de la psicología y de la ergonomía, se refiere a la compensación de perturbaciones a través de la búsqueda de nuevos equilibrios. En psicología se le conoce como un proceso dinámico enfocándose en las interacciones que permiten mantener un equilibrio entre un individuo y su medio externo (Piaget, 1975). Este re-equilibrio no consiste únicamente en el restablecimiento del sistema en su estado anterior, sino que también puede conducir a un nuevo equilibrio más adaptado a la situación. Éste se efectúa a través un doble proceso de asimilación y de acomodación. A largo plazo, ésta homeostasis no es solo reactiva sino que se vuelve también pro-activo, es decir que busca prevenir, anticipar las perturbaciones posibles y evitarlas.

En lo que se refiere a los riesgos, la regulación del sistema laboral cubre entonces los procesos de diagnóstico, de previsión, de anticipación, de evitación, de control y de recuperación. Esta regulación global es de hecho el resultado de múltiples regulaciones que corresponden al funcionamiento de diversos elementos constitutivos del sistema de orden económico, organizacional, cognitivo, fisiológico, biológico...

Con el fin de asegurar la fiabilidad del sistema, estos procesos de regulación implican una gestión individual y colectiva de las interacciones entre los operadores y su medio laboral, así como entre centros de decisión distintos.

\section{Las regulaciones colectivas : entre gestión vertical y horizontal del sistema socio-técnico}

11 En los sistemas laborales estudiados, la regulación colectiva es el resultado de una red jerárquica y funcional en la medida en que ésta es generada por esa red y al mismo tiempo es necesaria para su funcionamiento. Dicha red está constituida por actores situados en diferentes niveles jerárquicos, por las relaciones, intercambios y comunicaciones que éstos conllevan (De la Garza, 1999) así como por las intervenciones de los operadores. La figura 1 es un ejemplo de una red identificada a través del análisis de las actividades de los operadores del servicio eléctrico de la SNCF. Se ve como dichos operadores que se supone trabajan solos y muchas veces de manera "aislada" forman parte de una red funcional que influencia sus operaciones. Para ciertas operaciones, los operadores del servicio eléctrico dependen de centros de decisión de esa misma red los cuales pueden crear condiciones laborales no seguras si, por ejemplo no proveen a tiempo cierta información (cf. Figura 1, al final del artículo).

12 Se puede considerar que esta red se construye a través de une doble estructura, vertical y horizontal, en las cuales interactúan en momentos distintos del proceso laboral, los centros de decisión y operadores, cuyos objetivos laborales no sólo son diferentes sino que también pueden ser contradictorios. Esta doble estructura no es rígida ni completamente fija y se actualiza a través de las interacciones verticales y horizontales.

\subsection{La gestión vertical como sistema de interacciones ascendentes y descendentes entre diferentes trabajadores y centros de decisión}

13 La gestión vertical refleja la distinción oficial de los niveles jerárquicos y centros de decisión que dictan las regulaciones estructurales de la acción establecidas por la organización de toda compañía. Dicha gestión fija las reglas previas relativamente estables con referencia a tres aspectos del funcionamiento de una empresa. 
Los objetivos de la empresa, entre los cuales la eliminación o protección de los riesgos laborales y disfuncionamientos. Por ejemplo en el transporte ferroviario, la prevención de los riesgos de colisión y de impacto por una circulación férrea.

- Las tareas prescritas y de manera implícita lo que se espera que cada trabajador aporte rigen el funcionamiento de la empresa, la repartición de las obligaciones de resultado y la planificación del trabajo.

- Los instrumentos y equipos disponibles que se supone disminuyen la carga de trabajo (automatización de funciones) por un lado, y por el otro controlan la vigilancia de los operadores (sistemas de control de vigilancia automáticos en los trenes por ejemplo) (Valot, Weill-Fassina, Guyot \& Amalberti, 1995).

\subsection{La gestión horizontal como sistema de interacciones entre actores, equipos y oficios de un mismo nivel jerárquico}

15 La gestión horizontal tiene en parte un estatus menos oficial. Se refiere a las regulaciones operativas desarrolladas en la ejecución efectiva del trabajo para compensar los gajes del oficio. Se trata sobre todo de una gestión de los recursos tomando en cuenta los objetivos de la producción por los trabajadores implicados en un proceso laboral, cuales quieran que sean sus estatus, funciones, tareas $u$ oficios. Dicha gestión se puede entender a través del establecimiento de compromisos y de ajustes en un contexto laboral más o menos perturbado, difícil o con restricciones fuertes (fisiológicas, cognitivas, de producción, de riesgo, gestión del tiempo, etc.).

16 Estos aspectos implican adaptaciones, ajustes y la elaboración de nuevas reglas por parte de los operadores. Dichos aspectos no se refieren a digresiones o violaciones mayores del marco reglamentario, sino más bien a modos de compensación, que si bien son parciales, éstos son eficaces en la mayoría de los casos pues tienen como objetivo el asegurar un equilibrio entre practicas, restricciones, obligaciones, suponiendo a veces actividades autónomas y discrecionales con relación a lo previsto (Valot et al., 1995 ; De la Garza et al., 1999). Si algunas reglas operativas se establecen de manera previa, otras se construyen en la acción laboral a través de las motivaciones, experiencia, evaluaciones subjetivas de los riesgos, y posibilidades de adaptación de los operadores.

Esta gestión horizontal es más dinámica que la gestión vertical en el sentido en el que ésta responde y enfrenta los gajes del oficio a corto plazo y se actualiza en tiempo real en el curso de cada operación laboral, se modifica y evoluciona según las circunstancias y exigencias laborales. Sin embargo, dicha gestión no puede existir sin la gestión vertical y ambas pueden ser complementarias o antagónicas (De la Garza, 1999).

En esta gestión colectiva de los recursos se pueden distinguir dos modalidades de interacciones.

Interacciones cotidianas relacionadas con las situaciones laborales habituales que se construyen con el tiempo, la experiencia y gajes del oficio, sobre la base de relaciones de confianza, del conocimiento del otro y de sus capacidades. El desarrollo de tales interacciones favorece la creación de un "colectivo laboral" (Cru, 1995). Dicho colectivo laboral esta regido por "reglas del oficio" y un "referencial común" (de Terssac, 1992) que resulta de concertaciones implícitas en el ámbito laboral pero que pueden ser identificadas por medio de la observación de campo. 

trabajar juntos ante una situación critica o incidental. Es el caso por ejemplo de una red espontánea en un puesto de control del tráfico ferroviario presenciando una organización colectiva para recuperar un incidente ferroviario que bloquea el tráfico de una vía del tren (Weill-Fassina \& Bourdon, 1994). A ese tipo de red no se le puede anticipar y es por lo tanto mucho más difícil poder observarle de manera directa. Su análisis en general se puede establecer a partir de eventos que se produjeron anteriormente y de las consecuencias en la fiabilidad del sistema socio-técnico en relación con las acciones y decisiones realizadas.

Las modalidades de regulaciones tanto individuales como colectivas dependen a la vez de las características de cada operador y de su experiencia y posibilidades de acción según las exigencias de la tarea y restricciones de cada contexto laboral. Dichas posibilidades reflejan los espacios de libertad dejados de manera voluntaria o no por el sistema de reglas de una organización, creando así "zonas de tolerancia" relacionadas con las practicas aceptadas (Rasmussen, 1997). Las fronteras o umbrales entre lo aceptado o no aceptado pueden ser vagos y los limites se constituyen por la emergencia de incidentes durante el proceso laboral.

\section{Impactos del trabajo colectivo en la seguridad y la fiabilidad del sistema socio-técnico en dos situaciones laborales}

Partiendo del análisis de dos situaciones de trabajo colectivo en el ámbito ferroviario, vamos a ilustrar aquí cómo las posibilidades y modalidades de gestión temporal tienen una incidencia sobre la fiabilidad del sistema socio-técnico (De la Garza et al., 1999). Un resultado importante es que éstas dos situaciones sumamente distintas, presentan sin embargo puntos comunes que permiten así extrapolar los resultados y conceptos a otros procesos laborales.

\subsection{Las obras de las vías : un trabajo en equipos en zonas de "riesgo de impacto"}

En las obras de las vías del tren existen pequeñas obras de mantenimiento realizadas por equipos de agentes de vías de tres a máximo doce operadores. Estas obras consisten en el reemplazo de rieles, travesaños, mordazas, el chequeo de la geometría de las vías, la consistencia del balasto, etc. Para ese tipo de obras existen dos reglas esenciales previas sumamente estrictas para la seguridad del personal con el fin de evitar el riesgo de impacto :

- El bloqueo de la vía sobre la cual se realizan las obras o de la vía contigua a las obras. Esto necesita una información oficial y el establecimiento de una ficha entre el Departamento de mantenimiento y el de la explotación del tráfico ferroviario. Los tiempos de intervención son el resultado de compromisos entre las exigencias de las tareas de mantenimiento y las del tráfico ferroviario, con una prioridad por el tráfico.

- Una "protección humana" con un "anunciador" que consiste en un operador con un dispositivo sonoro para dar la señal unos segundos antes de la llegada del tren con el fin que el equipo se retire a tiempo y que el tren pase. El anunciador con el responsable de las obras 
decide en donde colocarse y del momento del anuncio del tren (a que distancia del equipo, que tiempo de anuncio, que visibilidad del tren). Esta decisión se toma en base a una tabla que da la distancia de "anuncio del tren" en metros, en relación con la "velocidad máxima" del tren y el "tiempo de anuncio" en segundos, según el tipo de obras y el material utilizado. Estas instrucciones emanan de una visión mecanicista del trabajo basada en la hipótesis de un comportamiento "estimulo-respuesta" (señal sonora - retiro "automático" del equipo). de los operadores según el entorno físico y climático con el fin de disminuir la incertidumbre y de aumentar el margen de seguridad, en particular en términos de tiempo. Intentan construirse representaciones mentales operativas de su entorno laboral durante las diferentes etapas de una tarea. Por ejemplo, al momento del comienzo de una obra, las instrucciones en "metros" para el establecimiento de la distancia de anuncio del anunciador es prácticamente imposible de llevar a cabo. Se trata entonces más bien de anticipar la detección del tren sobre la base de indicios visuales o auditivos del entorno laboral, así como de evaluar les tiempos probables de retiro del equipo de las vías de trabajo según su experiencia, operaciones de mantenimiento, estado del material, etc. Dichas evaluaciones toman en cuenta las posibilidades de percepción del anuncio sonoro por el equipo para poder retirarse a tiempo (por ejemplo el uso de equipo de trabajo excesivamente ruidoso necesita que un anunciador este justo al lado del equipo y otro más lejos, en un entorno nevado; el anunciador tiene que estar mucho más cerca del equipo para que este perciba el anuncio).

Este tipo de regulaciones requieren la construcción de una cierta "autonomía" por parte de los operadores teniendo como objetivo el anticipar el riesgo y el controlar el peligro de "impacto por un tren". La autonomía "se refiere a un espacio de libertad de decisión que todo actor individual o colectivo se construye y afirma en un sistema estructurado del exterior" ; significa la capacidad de producir sus reglas y organizar sus propios procesos de acción (Maggi, 1996 ; De la Garza et al., 1999). Desde luego que esto es imposible cuando se trata de novatos, la experiencia es un factor determinante de la eficacia de este tipo de regulaciones.

\subsection{La reparación de las instalaciones eléctricas de señalización ferroviaria : un trabajo individual inscrito en una red organizacional}

La reparación de las instalaciones de señalización ferroviaria es una de las actividades de los agentes del servicio eléctrico. Se trata de una intervención no prevista, cuyos modos operatorios son prácticamente imposibles de establecer dadas la naturaleza y la diversidad de las fallas, así como la diversidad de las instalaciones y de la red ferroviaria (reparación de los semáforos y otro tipo de señales, motores diversos, circuitos de las vías y otros, etc.). Desde el punto de vista de las reglas oficiales, el agente es el único responsable de su trabajo. Sin embargo, interviene en un marco regulado de acuerdo con el departamento de explotación para poder organizar su trabajo, realizar las instrucciones técnicas de seguridad y definir los tiempos de intervención. Además tiene por obligación entregar las instalaciones "a tiempo" y en

Laboreal, Volume 2 N²$^{\circ}$ | 2006 
"buen estado", es por eso que una etapa de "auto-control" al final de toda intervención esta prevista y dicho auto-control puede necesitar la colaboración del departamento de explotación, en particular el agente del puesto de regulación del tráfico. regulaciones estructurales y regulaciones operativas es sobre todo una gestión instrumental de recursos. La fiabilidad individual desde el punto de vista cognitivo depende de una fiabilidad colectiva facilitando ciertos recursos o al contrario integrando nuevas restricciones y reduciendo márgenes de seguridad. Se pueden constatar procesos de compensación más o menos parciales, compromisos que buscan disminuir la incertidumbre y paliar alguna falla del sistema organizacional, pero que al final ponen en peligro la fiabilidad del sistema socio-técnico (Fadier, De la Garza \& Didelot, 2003).

31 En la parte siguiente proponemos un marco teórico para el análisis del trabajo colectivo establecido a partir de los estudios de campo presentados. El interés es que dicho marco teórico puede utilizarse para el análisis de otras situaciones profesionales presentando un trabajo colectivo como recurso para el mantenimiento de la fiabilidad, de la salud o de la eficacia del sistema socio-técnico.

\section{Multi-dimensionalidad del trabajo colectivo : cuatro formas de interacciones sociales}

Con el fin de analizar el trabajo colectivo en todo proceso laboral, proponemos cuatro formas de interacción social que permiten distinguir la unidad de análisis del trabajo colectivo, los objetos de la acción, los resultados previstos, las tareas y los actores 
implicados (De la Garza \& Weill-Fassina, 2000 ; De la Garza, 1999). Las formas de trabajo colectivo evocadas aquí caracterizan secuencias distintas de la actividad laboral y pueden estar más o menos presentes durante la fase de organización o de realización. No se trata de alternativas y pueden referirse a la actividad de cualquiera de los actores de una empresa. Las definiciones propuestas no coinciden forzosamente con otras definiciones que existen en otros estudios ergonómicos o de psicología del trabajo o social. Sin embargo el interés de estas definiciones es que se construyeron a parir de un enfoque empírico para distinguir formas de trabajo colectivo de manera coherente que se observaron directamente en múltiples estudios de campo. Desde el punto de vista teórico, dichas definiciones fueron guiadas por las relaciones entre la actividad, la acción y la operación realizadas, yendo desde el término general hasta la transformación final en el proceso de producción. Se consideran unidades cada vez más finas de análisis de la conducta a partir de las cuales se puede analizar el trabajo colectivo. Las distinciones hechas toman en cuenta los resultados esperados, el espacio en el cual se realizan, los tiempos previstos, las operaciones de cada uno de los oficios implicados.

\subsection{Co-actividad, co-acción, cooperación y colaboración : cuatro conceptos para describir el trabajo colectivo}

- La co-actividad se observa en una situación en la cual dos especialidades diferentes (o más) trabajan juntas en un espacio común geográfico con objetivos de producción distintos, aún a largo plazo, salvo un objetivo de funcionamiento general de la empresa (Faverge, 1970). Por ejemplo en el ámbito ferroviario, en una zona delimitada protegida por un dispositivo de seguridad común - intercepción de una vía - dos equipos distintos trabajan de manera totalmente independiente, uno en la cuestión del ajuste del balasto y el otro en una obra de operaciones de señalamiento.

- La co-acción se refiere a situaciones en las cuales los operadores realizan acciones distintas sobre objetos distintos, tienen objetivos de producción a corto plazo distintos pero deben ser inscritos a medio o largo plazo en una actividad común. Es así como el reemplazo de una parte de un riel, necesita la participación de un soldador para soldar al final los dos pedazos de riel y de un equipo de agentes de las vías. Mientras que el soldador prepara los moldes, la soldadura, etc. los agentes de las vías serruchan el riel, desmontan las morazas y travesaños, etc. Al momento de la soldadura hay una actividad común ya que los agentes de vías tienen que hacer operaciones de ajuste de los rieles de manera simultanea.

- La cooperación caracteriza une actividad colectiva en la cual los operadores trabajan juntos en un mismo objeto o tienen el mismo objetivo final. La cooperación implica una repartición del trabajo según los conocimientos del proceso laboral, la experiencia, los recursos humanos, y las exigencias y restricciones laborales inmediatas. Dicha cooperación necesita disponibilidad temporal y personal de parte de los actores involucrados. Por ejemplo en las obras de las vías se observan actividades de cooperación cotidianas para levantar o reemplazar los travesaños, checar y nivelar el balasto, etc. En este tipo de proceso laboral se trata principalmente de la repartición de una fuerza física además de conocimientos técnicos. Pero se observa también una vigilancia colectiva entre el equipo de trabajo y el anunciador, que atestigua una cooperación mutua que tiende a aumentar la seguridad. Es efectivamente difícil mantener un estado de vigilancia optimo durante 6 horas seguidas en algunos casos! En otro tipo de procesos laborales la repartición podrá ser de tipo cognitivo, 
como es el caso en proyectos de diseño y de organización (de Terssac \& Friedberg, 1996 ; de Terssac \& Lompré, 1994).

- La colaboración significa una actividad colectiva en la cual los operadores ejecutan sobre un mismo objeto operaciones distintas que se articulan las unas con las otras y que tienen en general un objetivo común a corto o a mediano plazo. Dicha colaboración involucra diferentes unidades de trabajo o centros de decisión que no están forzosamente en el mismo espacio geográfico. Por ejemplo, antes de entregar las instalaciones, después de una intervención en algún dispositivo de señalamiento, todo agente del servicio eléctrico tiene que colaborar con un agente del departamento de explotación que se encuentra en un puesto de control del tráfico con el fin de checar y controlar el estado actual del dispositivo de señalamiento. Para esto, el agente del puesto de control tiene que hacer ciertas operaciones que le dicta por radio-teléfono el agente del servicio eléctrico. De este tipo de colaboración depende en parte la fiabilidad del sistema socio-técnico pues una confusión durante la comunicación con el agente del puesto de control puede inducir un error de cableado o de conexión eléctrica, dejando así un riesgo latente en las instalaciones eléctricas.

Otra forma de trabajo colectivo cooperativo que no observamos directamente pero que otros autores señalan es, por ejemplo en un comedor universitario, la "ayuda" entre operadores. Avila Assunção (1998) observó como ante una incapacidad de ciertas trabajadoras enfermas, algunas de las otras trabajadoras se organizan para ayudarlas a efectuar las tareas u operaciones difíciles o en algunos casos de plano realizan la tarea $u$ operación en lugar de la trabajadora enferma. Sin embargo, si este tipo de ayuda permite compensar las deficiencias de las trabajadoras enfermas y obtener los resultados esperados en términos de calidad y de producción, esto no es una situación deseable ni positiva, un problema de salud tal necesita una política de prevención.

\subsection{La concertación y la coordinación : dos condiciones necesarias para el funcionamiento del trabajo colectivo}

La concertación y la coordinación condicionan de cierta manera el trabajo colectivo facilitando o impidiendo las interacciones entre los diferentes actores implicados en todo proceso laboral.

- La concertación es un instrumento del trabajo colectivo para ponerse de acuerdo, para actuar juntos, lo que supone una confrontación y un ajuste de los puntos de vista de cada uno en cuanto por ejemplo a aspectos técnicos, de personal, de tiempos de trabajo. Cuando los actores implicados en un proceso laboral comparten un referencial común esta concertación es más fácil. Se observan situaciones en las cuales la concertación fracasa conduciendo a un conflicto entre actores cuyas tareas pueden ser dependientes del otro pero con objetivos de producción distintos y en ciertos casos incompatibles. Es así como los agentes del servicio eléctrico deben ponerse de acuerdo con los agentes del puesto de control del tráfico para organizar una operación, un chequeo, etc. Para el agente del puesto de control esto no es una tarea "asignada" y se le agrega a sus otras tareas, lo cual en ciertos periodos del día puede representar un problema (hora pico del tráfico ferroviario). Destaca entonces un proceso de negociación de parte de los actores con el fin de resolver el conflicto, sobrepasar las contradicciones del sistema y establecer compromisos (Touzard, 1987; de Terssac \& Friedberg, 1996). Ante la ausencia de un referencial común o de motivaciones compartidas, el conflicto tiene un rol estructurante aunque de cierto modo cada una de las 
partes trate de obtener un máximo de ventajas al final del compromiso. Por ejemplo varias de las interacciones entre los departamentos de mantenimiento y de explotación conciernen los lapsos de tiempo otorgados para las operaciones de mantenimiento. El agente del puesto de control intenta limitar dichos lapsos entre el paso de dos trenes para perturbar lo menos posible el tráfico, mientras que el agente de mantenimiento intenta darse cierto tiempo de maniobra al momento de la negociación anticipando algún imprevisto. Las interacciones funcionan en gran parte porque están fundadas en una relación de amistad o de confianza. Es raro el bloqueo total de la negociación y de la colaboración. Sin embargo cuando esto sucede, se operan cambios como por ejemplo una relación de poder y de estatus : un agente del servicio eléctrico que no logró obtener la colaboración del agente del puesto de regulación pasó el teléfono a su jefe el cual "ordena" el chequeo de las instalaciones eléctricas.

- La coordinación es la planificación y la organización temporal de las actividades. Implica un programa de conductas, acciones, decisiones y operaciones que se establece en términos de unidades temporales en relación con los recursos humanos y materiales (Valax \& Cellier, 1992). La coordinación se refiere a horizontes temporales diferentes sino es que divergentes ya que puede envolver diversos departamentos, especialidades, miembros de un mismo equipo según las etapas de un proceso laboral y las operaciones requeridas. Exige una compatibilidad en la temporalidad de las acciones e intervenciones según el programa previsto y los ajustes necesarios durante la realización efectiva del trabajo. Se distinguen entonces según Maggi (1996) coordinaciones previas, decididas en la estructura vertical que fija las reglas de toda intervención; se trata de coordinaciones diacrónicas y secuenciales que establecen los marcos temporales de la actividad futura. Después, durante la acción las coordinaciones contextuales caracterizan los modos efectivos de organización de los diferentes operadores y especialidades. Pueden ser secuenciales, simultáneas o de manera reciproca. Dicha coordinación tiene un carácter de eficacia y de gestión de la seguridad.

Estos conceptos han sido aplicados recientemente en un estudio de seguridad nuclear y se ha visto que funcionan mediante algunos ajustes necesarios dado el contexto particular (De la Garza \& Le Bot, 2006). En este caso han permitido demostrar una relación entre la fiabilidad, la eficacia y la eficiencia en el manejo de situaciones críticas en la sala de control.

\section{Conclusión}

Se muestra aquí como el trabajo colectivo puede tener un estatus diferente según las situaciones laborales : aparecer más bien como un criterio de eficacia y de fiabilidad o como un criterio de compensación de problemas de salud o de organización del personal. Luego entonces, Se le debe favorecer o modificar para mantener condiciones laborales adecuadas.

Este enfoque colectivo de las situaciones de alto riesgo se opone de cierto modo a un enfoque taylorista de la seguridad por varias razones.

- La seguridad no puede ser concebida como siendo el hecho o la responsabilidad de un solo individuo sino como el resultado de opciones y de decisiones que comprometen varios niveles de la empresa: el análisis de accidentes e incidentes laborales revela muy pocos casos de "lapsus" (Reason, 1990). La mayoría de los casos se inscribe en una multicausalidad implicando varios centros de decisión de la empresa así como distintas etapas de un proceso 
laboral, desde el diseño del equipo y puesto de trabajo en general, hasta la operación que efectuaba el operador o equipo, pasando por la organización del trabajo, etc.

- El accidente o incidente no puede ser juzgado como una digresión a una regla o norma, sino más bien como un intento de regulación que fracasó, sobrepasando los limites de adaptabilidad de los operadores. Dicho fracaso está a menudo ligado a restricciones de tipo temporal (urgencia, presión temporal, lapsos de intervención extremadamente cortos) que impiden la recuperación de un error o de una situación laboral que se degrada ante la imposibilidad de actuar o de actuar correctamente (falta de experiencia, falta de capacitación, etc.) (Reason, 1990 ; Rasmussen, 1997).

- Así, la seguridad no puede fundarse únicamente sobre diagnósticos de tipo de "hipovigilancia" o de "negligencia" por parte de un operador, como es el caso algunas veces en los reportes de accidentes laborales establecidos en las empresas. Este tipo de enfoque tiende a hacer del operador el culpable del accidente, mientras que éste es sólo el ultimo eslabón de una proceso de interacciones complejas, cuyo origen puede estar muy lejos.

- No se puede tampoco basarse en la hipótesis de una toma de riesgo ligada a la personalidad del operador ya que se trata en la mayoría de los casos de compromisos entre diversas exigencias del trabajo. El riesgo es evaluado por los operadores en función de ponderaciones diversas : resultados esperados, requerimientos de seguridad, de calidad, evaluación de sus propias capacidades y de las de los otros.

La prevención necesita en efecto un enfoque mucho más global articulando un punto de vista individual con un punto de vista organizacional.

Desde el punto de vista psicológico, la gestión colectiva del trabajo supone el conocimiento del otro, de sus capacidades y experiencia, de las estrategias y gajes del oficio para poder trabajar juntos de manera eficaz y en seguridad.

Desde el punto de vista ergonómico, este enfoque sobre la gestión colectiva del trabajo y de la seguridad se interroga sobre las posibilidades y los limites de una intervención en el ámbito de la prevención e higiene laboral. En efecto, una política de prevención debe considerar de manera global los riesgos laborales y problemas de salud, incluyendo diferentes centros de decisión de la empresa y no únicamente los “expertos" en seguridad. Una política de gestión global de la seguridad implica de hecho une gestión colectiva a niveles jerárquicos elevados así como la participación del personal de diseño de los equipos y puestos de trabajo, de diseño de reglas de seguridad, de organización de capacitación, de organización del personal, etc. (Hale \& Baram, 1998 ; Rasmussen, 1997) Esto fue mostrado recientemente en el marco de un proyecto europeo cuyo objetivo era el de proponer una metodología para el diseño de una organización segura en situaciones de conducta ferroviaria en Europa (De la Garza et al., 2004). 
Figura 1 - Red funcional del proceso laboral del agente del servicio eléctrico. El análisis ergonómico destacó interacciones entre servicios y departamentos de mantenimiento y de la explotación. Dichas interacciones se actualizan de manera distinta sobre la base de la estructura organizacional y de una red constituida en tiempo real y tienen un impacto en la seguridad cotidiana del sistema (De la Garza, 1999).

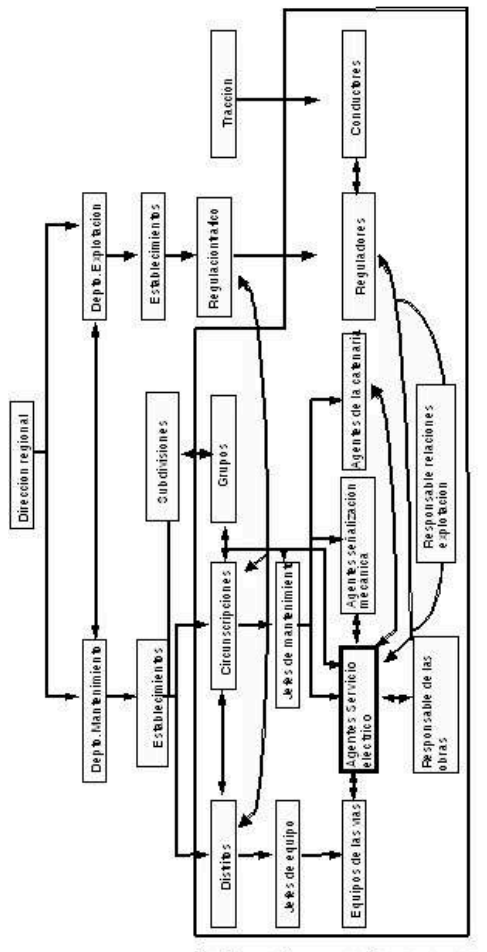

\section{BIBLIOGRAFÍA}

Avila Assunçao, A. (1998). De la déficience à la gestion collective du travail : les TMS dans la restauration collective universitaire. Doctoral dissertation, EPHE, Laboratoire d'Ergonomie Physiologique et Cognitive, Paris.

Baram, M. (June, 1995). Safety management : organizational learning disabilities in using incident analysis. Paper presented at Network Meeting, Bad Homburg, Germany.

Cox, S., \& Cox, T. (1996). Safety systems and people. Oxford: Butterworth-Heinemann.

Cru, D. (1995). Règles de métier, langue de métier : dimension symbolique au travail et démarche participative de prévention. Le cas du bâtiment et des travaux publics. EPHE Diploma, Laboratoire d'Ergonomie Physiologique et Cognitive, Paris.

De la Garza, C. (1999). Fiabilité individuelle et organisationnelle dans l'émergence de processus incidentels au cours d'opérations de maintenance. Le Travail Humain, 62 (1), 63-91.

De la Garza, C., \& Le Bot, P. (2006). Les simulations en conduite incidentelle/accidentelle dans le nucléaire : proposition d'un protocole pour l'analyse de l'activité collective. In L'humain comme 
facteur de performance des systèmes complexes, Actes du 10è congrès Ergo IA, 11-13 Octobre, Biarritz, France.

De la Garza, C., Maggi, B., \& Weill-Fassina, A. (1999). Tempo autonomia e discrezionalita nella manutenzione di infrastrutture ferroviarie/ Time, autonomy and discretion in railway maintenance. Ergonomia, 12, 36-43.

De la Garza, C., \& Weill-Fassina, A. (2000). Régulations horizontales et verticales du risque. In A. Weill-Fassina, T.H. Benchekroun (Eds.), Approches ergonomiques du travail collectif dans les systèmes socio-techniques (pp. 217-234). Toulouse : Octarès Éditions.

De la Garza, C., Weill-Fassina, A., Kaplan, M., Wilpert, B., Schmidt, A., \& Fahlbruch, B. (2004). Building a safe, interoperable railway : a methodological guide to integrating human factors. Université Paris 5 - EPHE - TU Berlin - UIC. Document published by the UIC, Paris, France.

De Montmollin, M. (1992). The future of ergonomics : hodge-podge or new foundation. Le travail humain, 55 (2), 171-181.

Fadier, E., De la Garza, C., \& Didelot, A. (2003). Safe design and human activity : construction of a theoretical framework from an analysis of a printing sector. Safety Science, 41 (9), 759-789.

Faverge, J. M. (1970). L'homme agent d'infiabilité et de fiabilité du processus industriel. Ergonomics, 13 (3), 301-327.

Hale, A, \& Baram, M. (Eds.) (1998). Safety management. The challenge of change. Oxford: Pergamon, Elsevier.

Hendrick, H.W. (1991). Ergonomics in organizational design and management. Ergonomics, 34 (6), 743-756.

Maggi, B. (1996). La régulation du processus d'action de travail. In P. Cazamian, F. Hubault, M. Noulin (Eds.), Traité d'ergonomie (pp. 637662). Toulouse : Octarès Éditions (3ème édition).

Piaget, J. (1975). L'équilibration des structures cognitives : problème central du développement. Études d'épistémologie génétique, vol. 33. Paris : PUF.

Rasmussen, J. (1986). Information processing and human-machine interaction. North-Holland : Elsevier Sc. Publischers.

Rasmussen, J. (1997). Risk Management in a dynamic society : a modelling problem. Safety Science, 27, 2-3, 183-213.

Reason, J. (1990). Human error. Cambridge : University Press. Terssac de, G. (1992). Autonomie dans le travail. Sociologie d'aujourd'hui. Paris : P.U.F.

Terssac de, G., \& Friedberg, E. (Eds.) (1996). Coopération et conception. Toulouse : Octarès Éditions.

Terssac de, G., \& Lompré, N. (1994). Coordination et coopération dans les organisations. In B. Pavard (Eds.), Systèmes coopératifs : de la modélisation à la conception (pp. 175-201). Toulouse : Octarès Éditions.

Touzard, H. (1987). Conflits et négociation. In C. Levy-Leboyer, J.C. Sperandio (Eds.), Traité de psychologie du travail (pp. 789-811). Paris : PUF. 
Valax, M. F., \& Cellier, J. M. (1992). Aides à l'organisation du travail dans

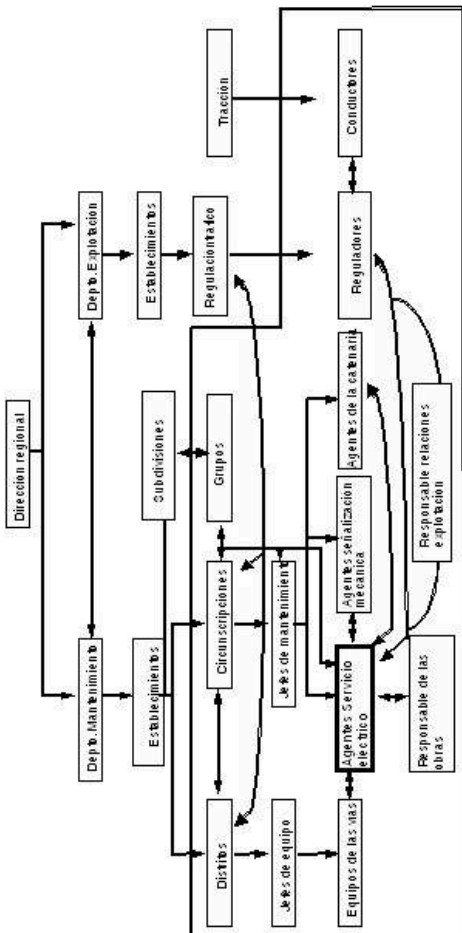

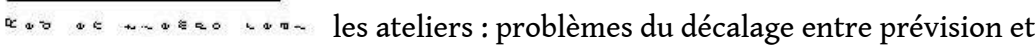

réalisation. In

G. de Terssac, P. Dubois (Eds.), Les nouvelles rationalisations de la production (pp. 121-137). Toulouse : Cepadues.

Valot, C., Weill-Fassina, A., Guyot, S., \& Amalberti, R. , (1995). Vers un modèle pour l'analyse des grands systèmes. Actes du XXXè Congrès de la SELF, Biarritz, France.

Weill-Fassina, \& A., Bourdon, F. (1994). Réseau et processus de coopération dans la gestion du trafic ferroviaire. Le Travail Humain, 57 (3), 271-287.

\section{NOTAS}

1. Ver número 1 de Laboreal para una presentación detallada de la técnica.

2. El término regulación se utiliza aquí en el sentido biológico y psicológico de Piaget (1975), como el conjunto de elementos y procesos que actúan para mantener constante un estado de un equilibrio (homeostasis).

\section{RESÚMENES}

Este artículo discute a través de una síntesis de análisis ergonómicos una serie de conceptos que guían el estudio del trabajo colectivo en procesos de alto riesgo en el ámbito ferroviario. Cuatro formas de trabajo colectivo fueron identificadas por la observación directa del trabajo efectuado 
por equipos de mantenimiento de las vías del tren y agentes del servicio eléctrico ferroviario. Se trata de la co-acción, la co-actividad, la colaboración y la cooperación, determinadas en parte por la coordinación y la concertación en el trabajo. La gestión de los riesgos de trabajo se caracteriza por articulaciones y regulaciones a nivel horizontal y vertical. Sin embargo, si el trabajo colectivo puede ser un criterio de seguridad del personal y del sistema así como un criterio de eficacia, los resultados muestran también los limites y ciertas lagunas a nivel de la seguridad. La organización de una prevención eficaz debe tomar en cuenta ese tipo de resultado.

Este artigo discute, através de uma síntese de análises ergonómicas, uma série de conceitos que guiam o estudo do trabalho colectivo em processos de alto risco no âmbito ferroviário. Quatro formas de trabalho colectivo foram identificadas, por observação directa do trabalho efectuado por equipas de manutenção da via ferroviária e por agentes de serviço eléctrico ferroviário. Trata-se da co-acção, da co-actividade, da colaboração e da cooperação, determinadas, em parte, pela coordenação e pela concertação no trabalho. A gestão dos riscos de trabalho caracteriza-se por articulações e regulações a nível vertical e horizontal. Contudo, se o trabalho colectivo pode ser um critério de segurança dos trabalhadores e do sistema, assim como um critério de eficácia, os resultados mostram também os limites e certas lacunas ao nível da segurança. A organização de uma prevenção eficaz deve ter em conta este tipo de resultados.

Cet article discute, par le biais d'une synthèse d'analyses ergonomiques, une série de concepts qui guident l'étude du travail collectif en procès de haut risque dans le domaine ferroviaire. Quatre formes de travail collectif ont été identifiées, par l'observation directe du travail efectué par des équipes d'entretien de la voie ferroviaire et par des agents de service électrique ferroviaire. Il s'agit de la co-action, de la co-activité, de la collaboration et de la coopération, en partie déterminées par la coordination et par la concertation dans le travail. La gestion des risques de travail se caractérise par des articulations et des régulations au niveau vertical et horizontal. Cependant, si le travail collectif peut être un critère de sécurité des travailleurs et du système, ainsi qu'un critère d'efficacité, les résultats montrent également les limites de certaines lacunes au niveau de la sécurité. L'organisation d'une prévention efficace doit tenir compte de ce type de résultats.

Ergonomic analyses of the railway field highlight concepts which guide the analysis of collective work. These concepts have been constructed through observation of two risky work processes in the field : rail tracks maintenance and maintenance operations in the electrical device of traffic regulation. Co-action, co-activity, collaboration and co-operation differentiate collective interactions which need coordination and negotiation. The occupational risk management is characterized by connections and regulations on horizontal and vertical levels. Nevertheless, even if ergonomic studies show how collective work should be a safety and a performance criterion they also show how some conditions and lack of safety cannot be recovered. An effective form of prevention should take into account results of this type.

ÍNDICE

Palavras-chave: trabalho colectivo, segurança, ergonomia, prevenção

Mots-clés: travail collectif, sécurité, ergonomie, prévention

Keywords: collective work, safety, prevention, ergonomics

Palabras claves: trabajo colectivo, seguridad, ergonomía, prevención 


\section{AUTORES}

\section{CECÍLIA DE LA GARZA}

EDF R2D - Département Management des Risques Industriels, 92140 Clamart Cedex France cecilia.de-la-garza@edf.fr

\section{ANNIE WEILL-FASSINA}

Laboratoire d'Ergonomie Physiologique et Cognitive 1, Av. Général de Gaulle École Pratique des Hautes Études, 41, rue Gay Lussac,75005 Paris France

awl@noos.fr 